\title{
RIRE1, a retrotransposon from wild rice Oryza australiensis
}

\author{
Kenichi Noma, Reiko Nakajima, Hisako Ohtsubo, \\ and Eiichi Ohtsubo* \\ Institute of Molecular and Cellular Biosciences, The University of \\ Tokyo, Yayoi 1-1-1, Bunkyo-ku, Tokyo 113, Japan
}

(Received 17 August 1996, accepted 4 July 1997)

\begin{abstract}
RIRE1 is a retrotransposon present in wild rice Oryza australiensis in an extraordinary number of copies, and only a portion of the LTR sequence has been determined previously. Here, we isolated and sequenced DNA segments of various portions of RIRE1, revealing that the sequences of LTR and the internal region were 1523 and $5277 \mathrm{bp}$ in length, respectively. The internal region shows homology with the pol region in copia, a Drosophila retrotransposon, indicating that RIRE1 is a copia-like retrotransposon. The internal region of RIRE1 contained an open reading frame coding for genes, gag, pro, int, $r t$ and $r h$, like copia and retroelements related to it. A clone screened from a library of the O. australiensis genomic DNA contained solo LTR, which was flanked by direct repeats of a 5-bp sequence. This suggests that RIRE 1 generates a duplication of the target sequence of 5 bp upon retroposition. We observed that many RIRE 1 members were nested by another RIRE 1 member. This indicates that these RIRE1 members have received another RIRE 1 to make an extraordinary number of copies in the $O$. australiensis genome without giving a deleterious effect on the growth of rice cells.
\end{abstract}

\section{INTRODUCTION}

Retrotransposons are mobile genetic elements, which make their copies by retroposition (for a review, see Bingham and Zachar, 1989). Structural and functional analyses of retrotransposons in Drosophila indicate that they are similar to proviruses, the integrated forms of retroviruses (for a review, see Varmus and Brown, 1989). Many retrotransposons in plants have been identified and characterized. Examples are Ta1-3 in Arabidopsis (Voytas and Ausubel, 1988), Tnt1 in tobacco (Grandbastien et al., 1989), del in lily (Smyth et al., 1989), PDR1 in pea (Lee et al., 1990), Wis-2-1A in wheat (Lucas et al., 1992; Murphy et al., 1992), Tos3-1 in rice (Hirochika et al., 1992), and BARE1 in barley (Manninen and Schulman, 1993). Homologues of some of these elements seem to be present ubiquitously in many kinds of plants (Flavell et al., 1992; Moore et al., 1991; Voytas et al., 1992; Hirochika and Hirochika, 1993).

Retrotransposons have long terminal repeats (LTRs), which are present as direct repeat sequences at their $5^{\prime}$ and $3^{\prime}$ ends. The LTR sequence is bound by $5^{\prime}-\mathrm{TG} \cdot \cdot$ and ..CA-3' and contains functional sites, such as enhancer, promoter, polyA signal, termination signal, etc. The internal region flanked by two LTRs contains an open reading frame(s), which is divided into two regions, gag and pol.

\footnotetext{
* Corresponding author.
}

gag is the gene encoding an RNA-binding protein, Gag, which encloses RNA transcribed from retrotransposons in the chromosome and forms virus-like particles. pol is the region which is highly homologous to that in some retroviruses and encodes several kinds of proteins, such as protease, integrase, reverse transcriptase and RNase $\mathrm{H}$, which are required for retroposition. A primer-binding site (PBS) exists in the region immediately downstream of one LTR at the 5 ' end, which has a complementary sequence to the 3 ' portion of tRNA encoded by the host. tRNA annealing to PBS works as a primer to initiate reverse transcription of RNA to synthesize (-) DNA strand. A polypurine tract (PPT) exists in the region immediately upstream of the other LTR at the 3 ' end, which is a site used to initiate synthesis of (+) DNA strand.

We have previously used a PCR method to analyze tandem repeat sequences in rice genomes, and found that the pOa4 sequence (511 bp in length), which has been reported as a tandem repeat sequence in wild rice Oryza australiensis (Zhao et al., 1989), is actually a segment of an interspersed DNA sequence (Nakajima et al., 1996). The sequences expanded from pOa4 show partial homology with the LTR sequences of BARE-1 and Wis-2-1A (Nakajima et al., 1996). This indicates that $\mathrm{pOa} 4$ and the sequences expanded from it are derived from a retrotransposon, which is named RIRE1 (for RIce RetroElement 1) (Nakajima et al., 1996). Copy numbers of LTR of RIRE1 have been determined to 
be about 7500 and 180 per haploid genome of $O$. australiensis and $O$. sativa cv. Nipponbare, respectively (Nakajima et al., 1996). In this paper, we report the entire nucleotide sequence of RIRE1 in O. australiensis, and show that RIRE1 is a copia-like retrotransposon with a long LTR. Many RIRE1 members with an insertion of another RIRE 1 member existed in the O. australiensis genome with an extraordinary number of copies of RIRE1. Significance of this result will be discussed.

\section{MATERIALS AND METHODS}

Plant DNA preparation. Total DNA was isolated from the leaves of 2 weeks-old seedlings of O. australiensis W1538 according to the method described previously (Ohtsubo et al., 1991).

PCR. PCR (polymerase chain reaction) was carried out as described (Tenzen et al., 1994). Primers used are listed in Table 1. They were chemically synthesized using DNA synthesizer oligo $1000 \mathrm{M}$ (Beckman). For cloning of the PCR products, they were ligated to $\mathrm{pCR}^{\mathrm{TM}} \mathrm{II}$ vector by using the Cloning Kit (Invitrogen) and the ligation product was transformed into Escherichia coli XL1-Blue MRF" cells

Table 1. Synthetic oligodeoxyribonucleotide primers used

\begin{tabular}{llr}
\hline \hline Primer $^{\mathrm{a}}$ & \multicolumn{1}{c}{ Sequence $^{\mathrm{b}}\left(5^{\prime} \rightarrow 3^{\prime}\right)$} & Position $^{\mathrm{c}}$ \\
\hline E1 & ATCCAACACAATGTGTTCTCT & $316-298$ \\
E5' & GTGCCATTAGTTGGTCTCA & $347-366$ \\
E6' & TGTTAGTGATATGCCCT & $1-17$ \\
E6 & ATCCCTTTGCCTCACGAT & $726-709$ \\
E7 & ggatccGGCTCTGATACCA & $1543-1525$ \\
E8' & TACGGACGCTTAAGTG & $891-906$ \\
E10 & CCAGGAACACTTGCAAGTA & $1467-1449$ \\
E12' & CTGTTGACGGCTCGGCTGAGTT & $804-825$ \\
E13 & CGTGATATCCAACATCTCC & $7-29$ \\
E14' & AACGATCTATCGGCCCTT & $1432-1449$ \\
KN5' & AGGGACTGCGTGGATATTT & $3132-3150$ \\
KN6 & AGCGTACGAGACATCTGG & $6178-6161$ \\
KN10 & CTGTTGGTGTGGCATCTC & $2597-2580$ \\
KN11' & CAGTAGCTCATAGTTGCAAG & $3750-3769$ \\
KN15 & CGAATATTCCAACTCCGAGA & $5702-5683$ \\
KN16' & GGTTTACTTAACAAGGCTATC & $2515-2535$ \\
KN17 & CCTTCTCAATCCTCTACTTC & $3808-3789$ \\
KN18 & CCATATCTACTAAAGTCATCG & $4414-4403$ \\
KN19' & GACCAATGAGCTCAACG & $4432-4358$ \\
KN20' & TCGATCCAGAATCGCCT & $5618-5634$ \\
\hline
\end{tabular}

a Primers with non-primed numbers hybridize to the strand corresponding to the RIRE 1 transcript. Primers with primed numbers hybridize to the complementary strand. A pair of primers E7/E8' were used to obtain clones pRN116 and 118 (see Fig. 2). Similarly, five pairs of primers, E14'/ $\mathrm{KN} 10, \mathrm{KN} 16$ '/KN17, KN11'/KN18, KN19'/KN15, and KN20'/ E13, were used to obtain clones pKN20 pKN29 (see Fig. 2).

b Lowercase letters indicate nucleotides of a linker.

c Numbers indicate coordinates to the RIRE1 sequence (see Fig. 4).
(STRATAGENE). The ampicillin-resistant transformants were selected on plates containing $100 \mu \mathrm{g} / \mathrm{ml}$ of ampicillin.

The frequency of mutations induced by PCR under the condition described above is 1 per $642 \mathrm{bp}$ (Tenzen et al., 1994).

Construction of genomic libraries and screening clones from libraries. A genomic library of rice were constructed as described by Sambrook et al. (1989), using Lambda EMBL4/EcoRI Vector Kit and Gigapack Gold Packaging Extract (STRATAGENE).

To construct a plasmid genomic library, total DNA from the O. australiensis was digested with BamHI, and the digested fragments were ligated with plasmid pUC118 (Vieira and Messing, 1987) which was also digested with BamHI. The ligated molecules were transformed into $E$. coli XL1Blue MRF" cells, and the ampicillin-resistant transformants were selected on plates containing $100 \mu \mathrm{g} / \mathrm{ml}$ of ampicillin.

Hybridization. The recombinant phage plaques or transformants were subjected to blotting and hybridization with the RIRE1 probe, pRN28, as follows: The recombinant phage plaques or transformants were transferred to a nylon membrane filter Hybond- $\mathrm{N}^{+}$(Amersham), as described by Sambrook et al. (1989). The filter was incubated at $65^{\circ} \mathrm{C}$ for $20 \mathrm{~min}$ in $4 \mathrm{ml} / 100 \mathrm{~cm}^{2}$ of a hybridization solution, consisting of $6 \times \mathrm{SSC}(0.9 \mathrm{M} \mathrm{NaCI}, 0.09 \mathrm{M}$ trisodium citrate), $5 \times$ Denhardt's solution $[0.1 \%$ Ficoll (Pharmacia), $0.1 \%$ polyvinylpyrolidone (Nakarai), $0.1 \%$ $(\mathrm{w} / \mathrm{v})$ bovine serum albumin (Seikagaku Kogyo)], 0.5\% (w/v) SDS, and $100 \mu \mathrm{g} / \mathrm{ml}$ of sonicated salmon sperm DNA (Sigma). The filter was soaked in $2 \mathrm{ml} / 100 \mathrm{~cm}^{2}$ of the hybridization solution containing ${ }^{32} \mathrm{P}$-labeled probe DNA, and incubated at $55^{\circ} \mathrm{C}$ for 12 to $18 \mathrm{~h}$. The filter so treated was washed two times in $2 \times$ SSC containing $0.1 \%$ SDS for 5 $\mathrm{min}$ at $55^{\circ} \mathrm{C}$ and $0.1 \times \mathrm{SSC}$ containing $0.1 \% \mathrm{SDS}$ for $10 \mathrm{~min}$ at $55^{\circ} \mathrm{C}$.

The DNA fragment used as probe was cut out of plasmid pRN28 and labeled with $\left[\gamma^{-32} \mathrm{P}\right] \mathrm{dCTP}$ (Amersham, $185 \mathrm{TBq} /$ nmol) using Random Primer DNA Labeling kit Ver. 2 (Takara).

Nucleotide sequencing and computer analysis of the sequences obtained. DNA sequencing was carried out by the dideoxynucleotide chain termination method (Messing, 1983; Sanger et al., 1977), using dye-labeled primers (-21M13, PR1) and ABI PRISM Dye Primer Cycle Sequencing Ready Reaction kit (Perkin Elmer) with AmpliTaq DNA Polymerase, FS, or using dye-labeled terminator DyeDeoxy Terminator Cycle Sequencing kit with AmpliTaq (Perkin Elmer) and using relevant oligodeoxyribonucleotides primers (see Table 1). Heat-denatured plasmid DNA were used as templates. Sequencing reaction was carried out using Catalyst A800 (Perkin Elmer) and the reaction products were run using ABI 373S-36 DNA Sequencer. 
Alignments of sequence data were carried out using HarrPlot 1.2.2 and GENETYX-MAC 7.3 programs. A search for homology of nucleotide and amino acid sequences was performed by running the programs FASTA and BLAST against the sequences in GenBank and EMBL.

Accession number. The nucleotide sequence data reported will appear in the DDBJ, EMBL and GenBank nucleotide sequence databases under the accession number D85597.

\section{RESULTS}

Determination of the entire nucleotide sequence of RIRE1. We have previously shown that pOa4 is the sequence not repeated in tandem, but is a portion of LTR of a retrotransposon (named RIRE1) interspersed in the $O$. australiensis genome. In the previous experiments to show this, we carried out PCR using a pair of primers, Oa4b and Oa4f, which hybridize to the pOa4 sequence and prime DNA synthesis in one and the other directions, respectively (see Fig. 1), and obtained the fragments containing the 5' portions of the LTR sequence (see representative clones, pRN11, pRN17 and pRN28 shown in Fig. 2). Note that these fragments are derived from the RIRE1 members with an insertion of another RIRE1 member at different positions in the LTR sequence of RIRE1, as shown in Figure 1.
To determine the 3' portion of the LTR sequence of RIRE1, we obtained a clone, pRN134, by screening from a phage library containing Bam $\mathrm{HI}$ fragments of the O. australiensis genomic DNA using pRN28 containing a 5' portion of LTR

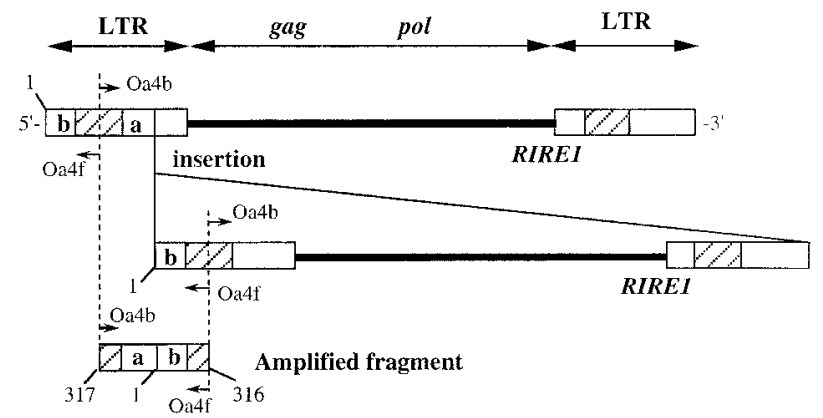

Fig. 1. Schematic representation of the structure of RIRE1 and a model for amplification of an RIRE1 segment by PCR. Positions of LTRs and internal regions containing gag and pol of RIRE1 are indicated at top. An RIRE1 element with an insertion of another RIRE1 element at a site within an LTR sequence is shown. Note that the RIRE 1 complex has region $\mathbf{a}$ in an LTR which is connected with region $\mathbf{b}$ in another LTR, as shown. PCR using a pair of primers which hybridize to the sequences in the pOa4 sequence (shadowed boxes) in LTRs can amplify a fragment shown at the bottom. Arrowheads indicate primers (Oa4f and Or4b) used to amplify the fragment from total DNA of $O$. australiensis (Nakajima et al., 1996). Numbers are coordinates given to the consensus sequence of RIRE1 (see Fig. 4).

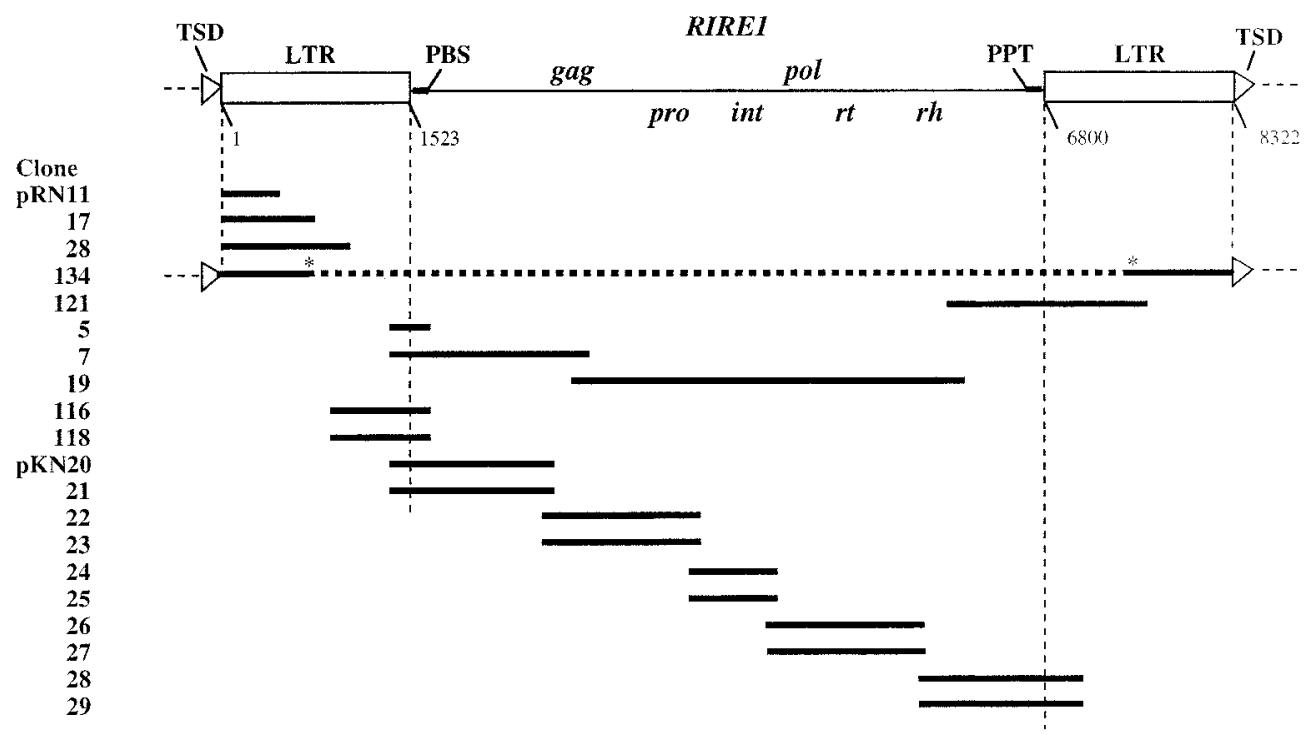

Fig. 2. Schematic representation of the structure of RIRE1 and clones containing various portions of RIRE1. Thick lines indicate portions of RIRE 1 carried by the clones. pRN134 carries an LTR sequence, which cannot be assigned to the sequence at the left or right side of RIRE1; therefore, the LTR sequence in pRN134 is indicated by two thick lines, which are separated by a dotted line, but are actually connected at sites indicated by asterisks in this figure. Numbers along the structure of RIRE 1 are coordinates to the RIRE1 sequence (see Fig. 4). TSD (open triangles), target site duplication of the sequence 5'-GGTCG-3'; LTR (open boxes), long terminal repeat; PBS, primer-binding site; PPT, polypurinte tract; gag, gag region; pol, pol region; pro, aspartic protease gene; int, integrase gene; $r t$, reverse transcriptase gene; $r h$, RNase $\mathrm{H}$ gene. 


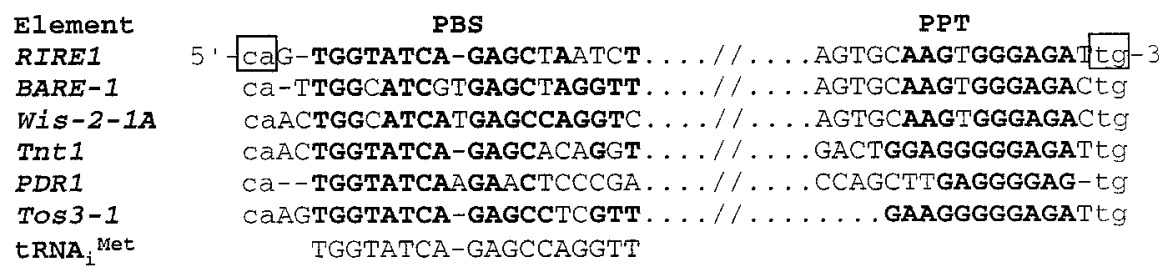

Fig. 3. Primer-binding sites (PBS) and polypurine tracts (PPT) in the internal regions of retrotransposons. The complementary sequence to the $3^{\prime}$ region of methionyl tRNA ( $\left(R N A_{i}{ }^{\text {Met }}\right.$ ) which initiates (-) strand DNA synthesis is also shown. Two di-nucleotide sequences of RIRE1 that are boxed and indicated by lowercase letters are of the LTR sequences, which are immediately adjacent to the internal sequence (see Fig. 2). These sequences are also present in other retrotransposons as shown. Boldface letters indicate PBS and PPT sequences in each retrotransposon.

as probe (see Fig. 2). Comparison of the sequence of pRN134 with that in pRN28 and with those of the other clones to be described below revealed that pRN134 contained solo LTR bound by 5 '-TG $\cdot$ and ' C CA-3' without the internal region of RIRE1 (Fig. 2). The solo-LTR sequence was flanked by direct repeats of a 5-bp sequence, 5'-GGTCG3' (see TSD in Fig. 2)

We also obtained another clone pRN121 by screening from a plasmid library containing $B a m \mathrm{HI}$ fragments of the $O$. australiensis genomic DNA using pRN28 as probe. Nucleotide sequencing revealed that $\mathrm{pRN} 121$ contained not only the 5' portion of LTR, which may correspond to that present at the right side of RIRE1 (see Fig. 2), but also PPT (Fig. 3). As described below, this clone also contained the 3 portion of the $r h$ gene in the pol region (see Fig. 2).

To determine the entire internal region of RIRE1, we carried out PCR to clone the fragments containing the PBS region adjacent to LTR at the 5 ' end of RIRE1 using a pair of primers (E13, E14'; Table 1), which hybridize to the end regions of LTR and prime DNA synthesis towards outside of the sequence. This is based on the assumption that if RIRE1 members with an insertion of another RIRE1 member exist in the internal region as depicted in Figure 1, the fragments containing the PBS region could be amplified by PCR using the primers described above. Nucleotide sequencing of several clones obtained revealed that two clones (pRN5 and pR N7; Fig. 2) contained PBS, which showed homology with the PBS sequences in other plant retrotransposons and contained a complementary sequence to the 3'end region of tRNA ${ }_{i}^{\text {Met }}$ (Fig. 3). There existed the putative gag region downstream of PBS (Fig. 2). We then carried out PCR using a pair of primers (KN5' and KN6'; Table 1), which hybridize to the sequences at the 3 ' end of pRN7 and at the $5^{\prime}$ end of pRN121. Sequencing of a clone pRN19 (Fig. 2) obtained revealed that it contained the pol region of RIRE1, leading to determination of the entire nucleotide sequence of RIRE1.

To confirm the entire sequence of RIRE 1 determined above, we carried out PCR using relevant pairs of primers, which hybridize to appropriate sites in the RIRE1 sequence (Table 1), and obtained clones containing DNA segments of
RIRE1 (pRN116, pRN118, and pKN20 29; Fig. 2). Nucleotide sequencing of these clones revealed that they contained RIRE1 sequences with a sequence difference, almost by base substitutions. From the sequences obtained, we could derive a consensus sequence of LTR and the internal region of RIRE 1, 1523 and $5277 \mathrm{bp}$ in lengths, respectively (Fig. 4). Comparison of the nucleotide sequences of the clones with the consensus sequence revealed that a few base changes, mostly by substitutions, occurred at a frequency of $1.1 \sim 3.4 \%$, which is higher than the frequency (about $0.2 \%$ ) of mutations induced by PCR under the condition used in the present experiment. This suggests that the clones obtained are derived from several copies of RIRE1 interspersed in different loci. Note here, however, that in the pol region, which is conserved well among retrotransposons, RIRE1 showed sequence homology of about $48.2 \%$ with that in copia, a Drosophila retrotransposon (Fig. 5A), and of about $66.5 \%$ with that in BARE1, a copia-like retrotransposon in barley (Fig. 5B). These numbers are much lower than that (about 98.5\%) of any one of the clones containing the pol region. Note also that more than $72 \%$ of the base substitutions observed in the internal coding region did not change amino acids or changed to conservative amino acids. We, therefore, believe that the consensus sequence obtained is the master sequence of RIRE1, from which many copies of RIRE1 have been originated.

Coding region. The internal region $(5277 \mathrm{bp})$ contained one large open reading frame, which could code for genes, gag, pro, int, $r t$, and $r h$, encoding Gag protein, protease, integrase, reverse transcriptase, and $\mathrm{RNase} \mathrm{H}$, respectively, in this order (Figs. 2 and 4). This confirms that RIRE1 is related to copia and the copia-like retrotransposons, which encode such genes in the same order and produce their proteins as a polyprotein.

The gag gene encodes an RNA-binding protein, Gag, which has been demonstrated to mediate positioning of the tRNA on (-) strand of retroviral RNA and initiating reverse transcription (Prats et al., 1988). There exists amino acid motif C (2) $\mathrm{C}$ (4) $\mathrm{H}$ (4) $\mathrm{C}$ which is conserved in a large family of nucleic acid-binding proteins (Berg, 1986). In 
1 ITR

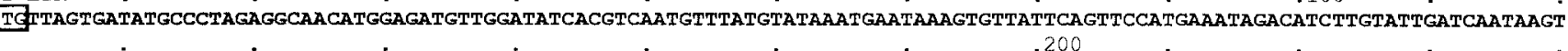

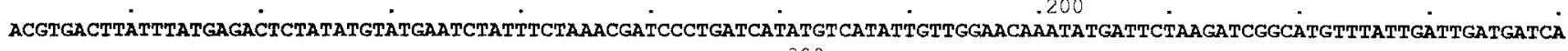

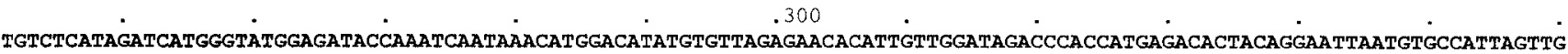

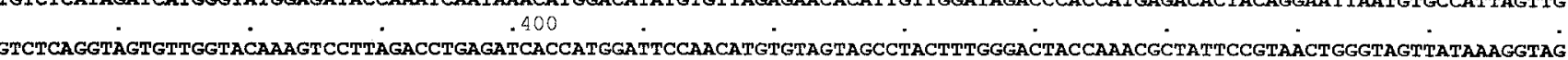
$500 \quad 600$.

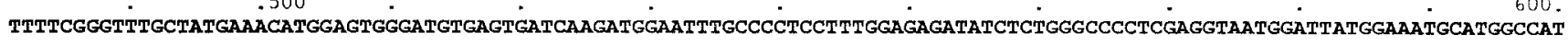

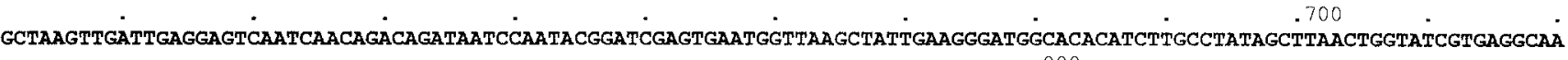

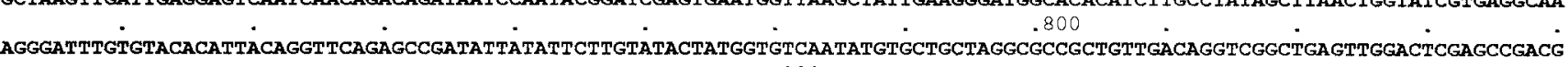

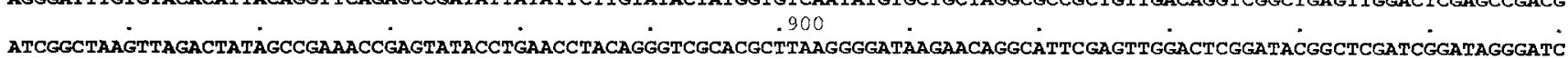
ATCGGCTAAGTTAGACTATAGCCGAAACCGAGTATACCTGAACCTACAGGGTCGCACGCTTAAGGGGATAAGAACAGGCATTCGAGTTGGACTCGGATACGGCTCGATCGGATAGGGATC CGATCGgATAGAgTCCTATGGGCTTACGACGTATGGGCGGCCAACTCTATGAGATACGGATGAGATCCGATTCAAAATAGAGTCCTGATCGGCCACTAGTCCCAGTCGGCCAACTCTATA $\begin{array}{rl}.1100 & 0\end{array}$ CGTGCTAGCACACGGGTGTTCGGCGTTCTGTCCCCGGACGTGTGGATACCGGTAGAGGCGCTGCTACGTTGCACGCTGTTGATCGGCTGCGGATCGGCTACGACATCCGCGAATCGGCTG ATTGGTGAATCGGTIGTCCCCGGGTATCGGCTGTCGGCTTGCGGGAATCGGCTACACTGTTCCGGATCGGATAGCCCTGATTGGCTATITCCGCTGCATACTCCAATCGGTAACGATCTA

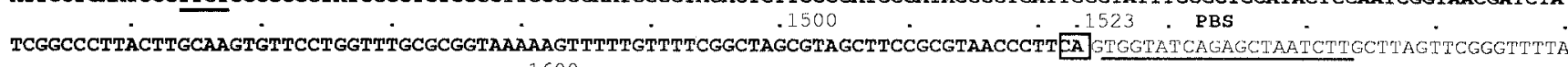

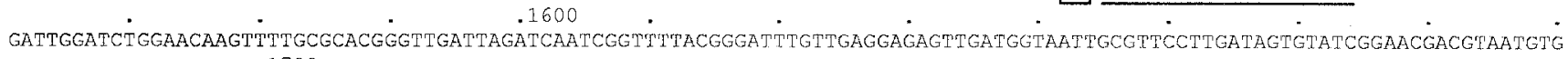

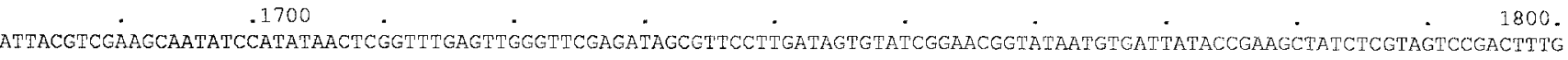

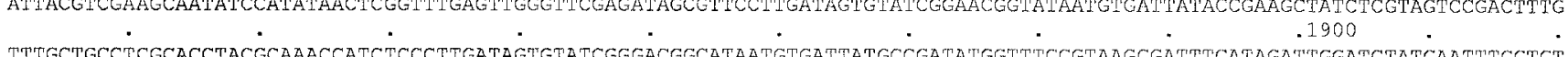
TTIGCTGCCTCGCACCTACGCAAACCATCTCCCTTGATAGTGTATCGGGACGGCATAATGTGATMATGCCGATATGGT'TCCGTAAGCGATTTCATAGATTGGATCTATGAATMTGTET GGTGTTACGGGGCCGTGTGCGTACCCCCITATAGGTCGTCTCGGTGCCGTATCTCTGCGATAGGTAGAAAACGTTTCTACGCCTAACTTGTTTTTGCAGAGAATGCTTPGAGATGGTAT" GGCCCTATTGTATGTATGAGATGCATGTGATGCATGTAATATTCTTTCATATGTGCAATCCCTGTAATATTCTTTCATACGTGCAATGCTTGTAATATTCGTTCACGACCTGCGAGTCGA TGTAATCTTCAATAGAGCTTCTATAGTAGTAGCTTAGTTTGAAGAAATCAAGCATTCGGCACTCGGAAGAACGGTGATGAAGATGGAGATCCTCTACACCGGCATGGAGATGGRGATCA .2300 . .2 .0 .2400$. CCATGTGAAGGGGGCCATACTATTTCATTATATGCTATPCTACTTGCTTTCATATATGTGATATGTTTGTTTGATAGGATAGCATCCCTCGCAAAATTAAGIAGTAATGATGCCCIPCCA AATGTTGCACCCGTCACCGPTATGCTCGTCAATGGTGGTCTGCCAAAGCAGAGTGCCATTCTCTATCATAACACGAGGGGGTATATGTCAGACATATRCATGCAGGAGCATETGGITIA CTTAACAAGGCTATCAAAACAGTTTGGGCCTTGGGGCATAGGTTGGCGCCGGGGCATGGAGATGCCACACCAACAACAAGAGTCACATAGAGATGTGATTAGCAAGGTGTTGCTTACCGG

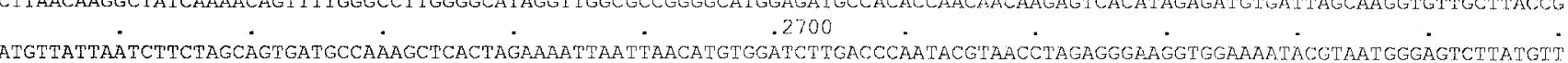

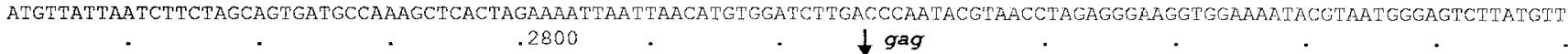

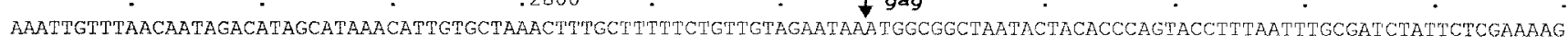

.2900

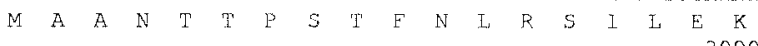
GAAAAATTAAATGGAACAAACTTIATGATTGGTATCGCAACTTGAGAATTGTTCTCAAGCAAGAGCGTAAGGAGTACGTTCTTGAAGTACCCTAICCTGAGGAGTTGCCTAATAATGCC

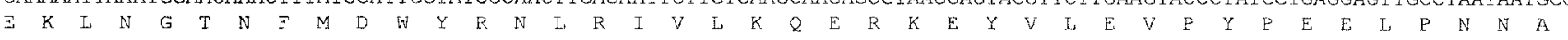
ACTGCTACTGCACGGAGGGCTTACGAGAAGCACACTAATGATGCCCTAGATATAAGCTGTCTCATGCTAGCTACAATGTCCCCTGAGCTTCAGAAGCAATATGAGAGCAGCGATGCTCAC

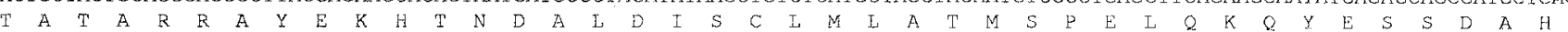
ACTACTATTCAGGGACTGCGTGGTATGTTTGAGAACCAAGCTCGgGACGAGAGGTTCAACACCTCAAAGTCCTTGTTTGCGTGCAGGCTTGTTGAGGGGAATCCCGTCAGTCCGCATGTG

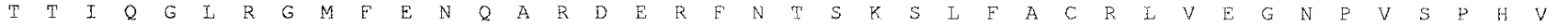

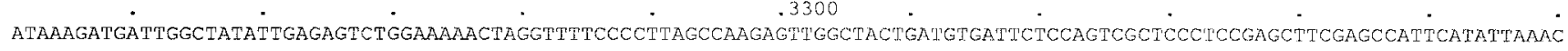

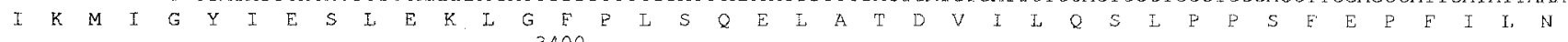
TATCACATGAACAATATGGATAGAACCTTGGCTGATTGCATGGGATGCTAAAGACAGCTGAGGAGAGTATCCAGAAAAATGGTCATCATGTGATGATGATGCAGAAGGCIAAGCGCAAA

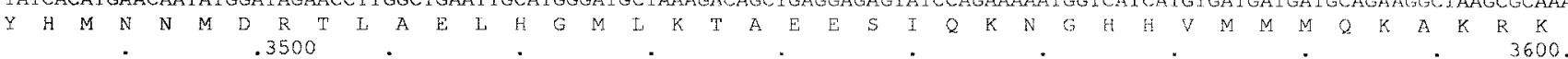
CCACCTGTCAAGAAACTTTGCACCAAGAGGAAGTTAACTCCCGATGAGATCGCGAGTGCCTCTAATGCAAAGAAGGGCAAGAAGGGGTCGCCGGCATCAGATGCCGTTTGCTTCTATTGC

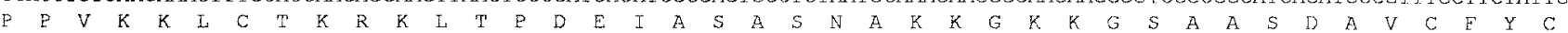
AAGGAGACAGGCCACTGGAAGAGGAACTGCAAGAAGTACATGGAGGATCTCAAAAAGAAGCAAAGTACGACTTCTGCTTCAGGTATTAATGTTATAGACATTAATCTIGGTACTTCACCT

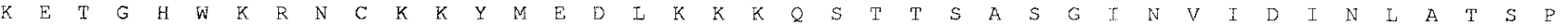
ACTGACTCTTGGGTATTTGATACCGGATCAGTAGCTCATATTTGCAAATCGTTGCAGGGAATGAGAAGAAGTAGAGGATGAGAAGGGGCGAGGTGARCCTGCGCGTCGGCAATGGAGCA

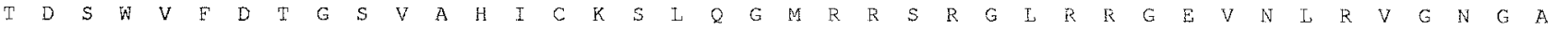
AGCGTTGCTACAGTTGCTGTCGGCACAGTACCACTTCATCPACCTICAGGATTAGTTTGGAATTGAATAATGTTATTGIGIPCCAACACTATGTCAAAACGTTATTTCCGCTTCATGT

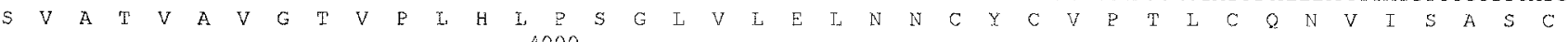
TTGCAAGCGGAAGGATATGATTTTAGATCAATGAACAATGGTTGTCAATATACCTCAGAGATATGTTCTATTTTCATGCTCCATTGGTGATGGATATACGTITIGAATCTPGAGCG

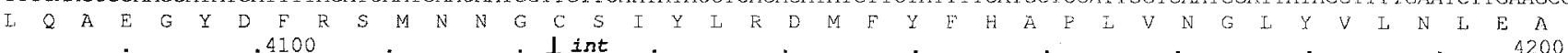

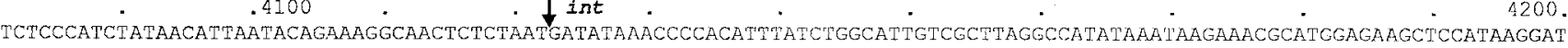

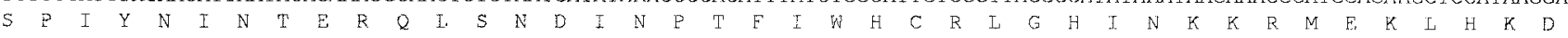


GGATTGCTTCACTCTTTTGATTTGAATCATTTGAGACATGTGAGTCTTGTTTACTTGGTAAGATGACAAAGGCACCTTTCACGGGACATAGTGAGAGAGCAAGTGACTTATTGGCACTC

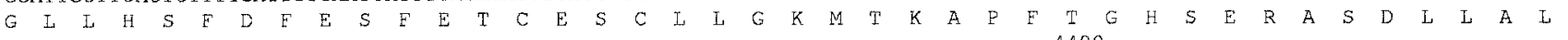

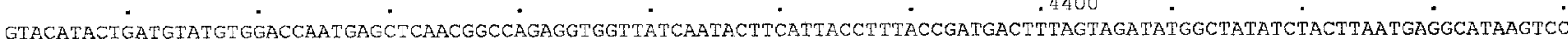

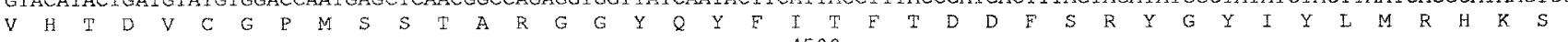
GAATCCTTTGAAAGTTCAAAGAATTCCAGATGAAGTACAGAATCATTTAGGGAAAACAATCAAGTTICTACGATCAGACCGTGGAGGGGAATACGTGAGCCAAGAGTTTGGTAATCAT'

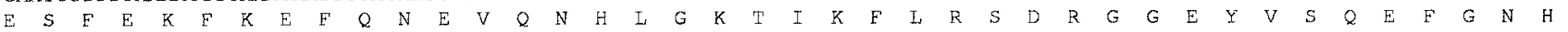
CTGAAGATTGTGGAATGTTCCACAGTTGACTCCGCCAGGAACTCCACAATGGAACGGAGTGTCCGAACGGAGAAATCGCACCTTGTTGGACATGGTGCGGTCGATGATGAGCCAAAGT

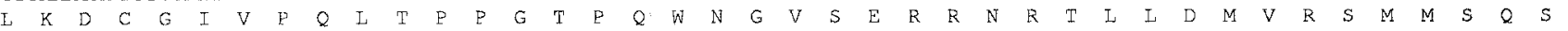
GATCTICCGTTATCCTTCTGGGGATATGCTCTTGAAACAGCTGCGCTCACACTAAATAGAGTTCCATCTAAGTCAGTTGAAAAGACACCATATGAGATATGGACAGGGCAACCCCCTAGT

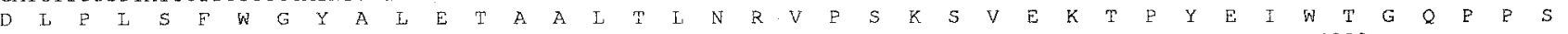
TTGTCTTPTCTCAAAATTTGGGGTGTGAGGCTTATGTAAAACGTTTACAATCTGACAAGCTCACACCCAAATCTGACAÄGGCTTCGTTGTGGGATATCCTAAGGAAACTAAGGGATAT

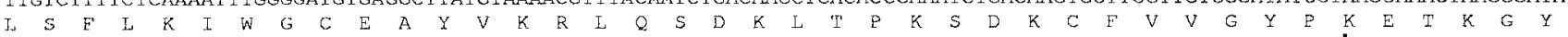

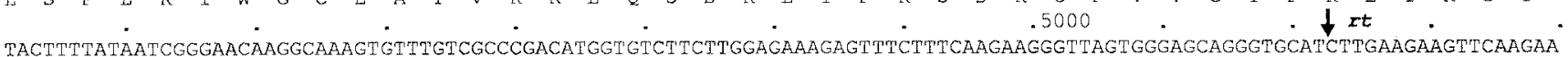

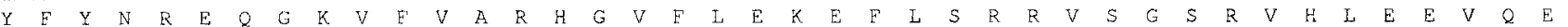
ACACCAGAAAATGTTTCAGCGACCACAGAACCACAACAGGAGGACCAAAGTGTTGCGCCACCAGTTGTAGATACACCAGCCCCACGGAGGTCTGAAAGATCACGTCGTGCGCCIGACAGG $\begin{array}{llllllllllllllllllllllllllllllllllllllllllllll}T & P & E & N & V & S & A & T & T & E & P & Q & Q & E & D & Q & S & V & A & P & P & V & V & D & T & P & A & P & R & R & S & E & R & S & R & R & A & P & D & R\end{array}$ TACACAGGTGCGGAACAACGTGATATATTGTTGTTGGACAACGATGAACCTAAGACCTATGAGGAAGCGATGGTGGGACCCGATTCCAACAAGTGGCTTGGAGCCATGAAAATCCGAAATA

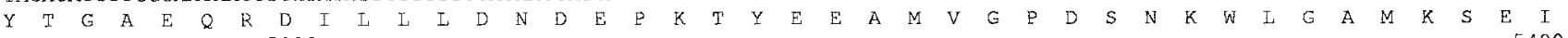
GAATCCATGTACGACAATCAAGTTGGAACTTGGTIGATCCACCTGATGGTGTCAAAACCATTGAGTGTAAATGGGTTTMTAAGAAAAAGGCCGATATGGATGGAAATGTTCACATCTAT

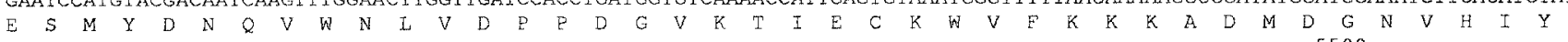
AAGGCGCGATTGGTGGCGAAAGGTTTTAAÄAGATTCAAGGAGTTGATTATGATGAAACCTTCTCGCCCGTCGCAATGCTTAAATCTATTCGGATTATCCTCGCGATTGCTGCATATTTC

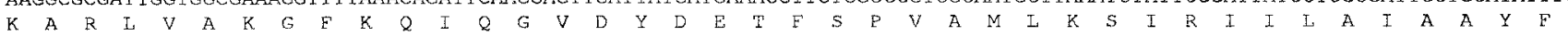
GATTATGAGATATGGCAGATGGATGTCAAAACGGCTTTCCTAAATGGAAACCTAAGCGAGGATGTATACATGATACAACCTCAGGGTTTTGTCGATCCAGAATCGCCTGGAAAGATATGC

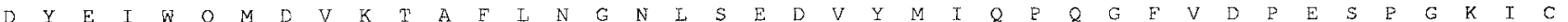
AAGCTACAGAAATCCATTTÁTGGATTGAAGCAAGCATCTCGGAGTTGGAATATTCGTTTTGATGAAGTAATCAAAGGGTPTGGTTTCATCAAAAACGAAGAAGAGGCCTGTGTTTACAAA

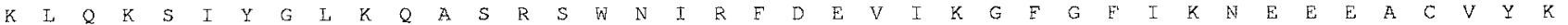
AAGGTCAGTGGGAGCGCAATTGTATTTCTAATCTTATATGTGGATGACATATTACTGATTGGAAATGATATCCCTATGCTAGAATCCGTCAAGTCTTCATTGAAAAATAGTTTTTCCATG

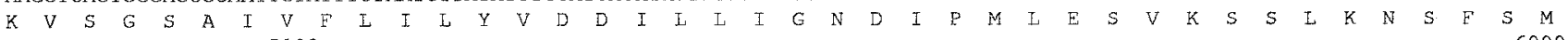
6000 AAAGACTTAGGGGAGGCAGCATACATATTGGGCATTCGGATCTATAGAGATAGATCCAAGAGGCTAATTGGATTAAGCCAAAGTACATACATTGACAAGGTGTIGAAAAGGTTCAACATG

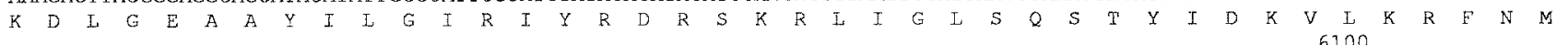
CATGATTCCAAGAAAGGTTCTTGCCCATGTCACATGGCATTAATCTTAGCAAGAATCAGTGTCCTCAGACACATGATGAGCGGAATAAGATGGGTATGGTTCCATATGCTTCGGCAATT

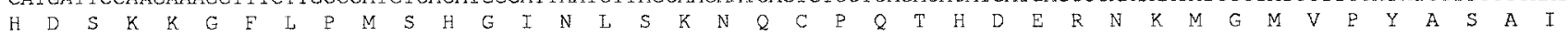
GGATCCATCATGTATGCCATGCTTTGTACACGCCCAGATGTCTCGTACGCTTTGAGTGCTACGAGCAGATACCAGTCAGATCCAGGTGAAGGTCACTGGACTGCCGTAAAGAATATCCTT

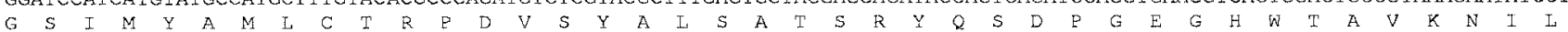

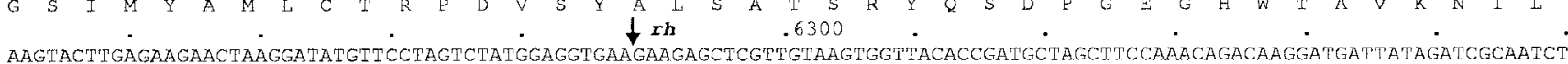

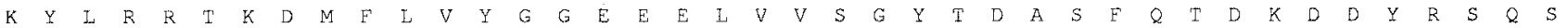
GGGTTCGTGTTCTGCCTGAACGGAGGCGCAGTCAGCTGGAAGAGTTCCAAGCAGGATACTGTTGCTGATTCTACAACGGAGGCCGAGTACATTGCTGCTTCGGAAGCTGCAAAGGAGGCT

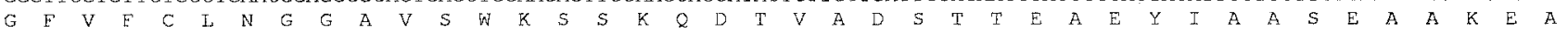
6500 . . . 6600. . . . . . . . . . GTTGGATCAAGAAATTCGTTCTGAGCTTGGTGTGATGACTAGTACGACTGGTCCAATGTCTCTCTATTGTGATAATAGTGGAGCCATTGCGCAAGCCAAGGAGCCGAGGTCACATCAG

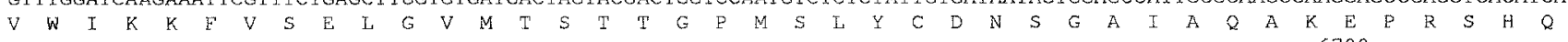
AAGTCCAAACACATACTTCGCCGATATCATCTCATCCGCGAGATAGTGGACAGAGGTGATGTCAAGATATGCAAAGTGCACACGGATCTCAACATAGCCGATCCGCTGACAAAACCTCTC

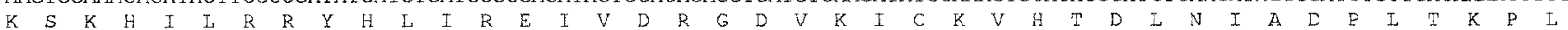

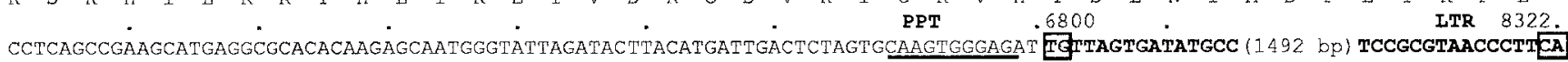

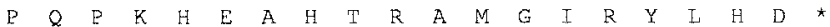

Fig. 4. The nucleotide sequence of RIRE1. This sequence is a consensus sequence, which was derived from the sequences of the fragments in the clones shown in Fig. 2. Numbers are coordinates given to the RIRE1 sequence. Boldface letters indicate the LTR sequences, in which the LTR sequence at the $3^{\prime}$ end of RIRE1 is supposed to be the same or almost identical to the LTR sequence at the 5 ' end, and the middle region of LTR at the 3 ' end is thus abbreviated in this Figure. Two di-nucleotide sequences, which are commonly present at the ends of LTR of retrotransposons, are boxed. PBS and PPT sequences are underlined. The other sequences underlined in LTR are explained in the text. Vertical arrows indicate start sites of genes, gag, pro, int, $r t$ and $r h$. See a schematic representation of the structure of RIRE1 shown in Fig. 2.

RIRE1, the coding region corresponding to gag existed at positions from 2827 to 3675 (Fig. 4) and contained this motif (Fig. 6).
In the pol region, the pro gene encodes an aspartic protease which cleaves the polyprotein. An amino acid sequence D-T-G in proteases is suggested to be an active site 
(Pearl and Taylor, 1987; Skalka, 1989; Lapatto et al., 1989). The coding region corresponding to pro existed in RIRE1 at positions 3676-4122 (Fig. 4), and contained this amino acid sequence (Fig. 6). The int gene encodes an integrase

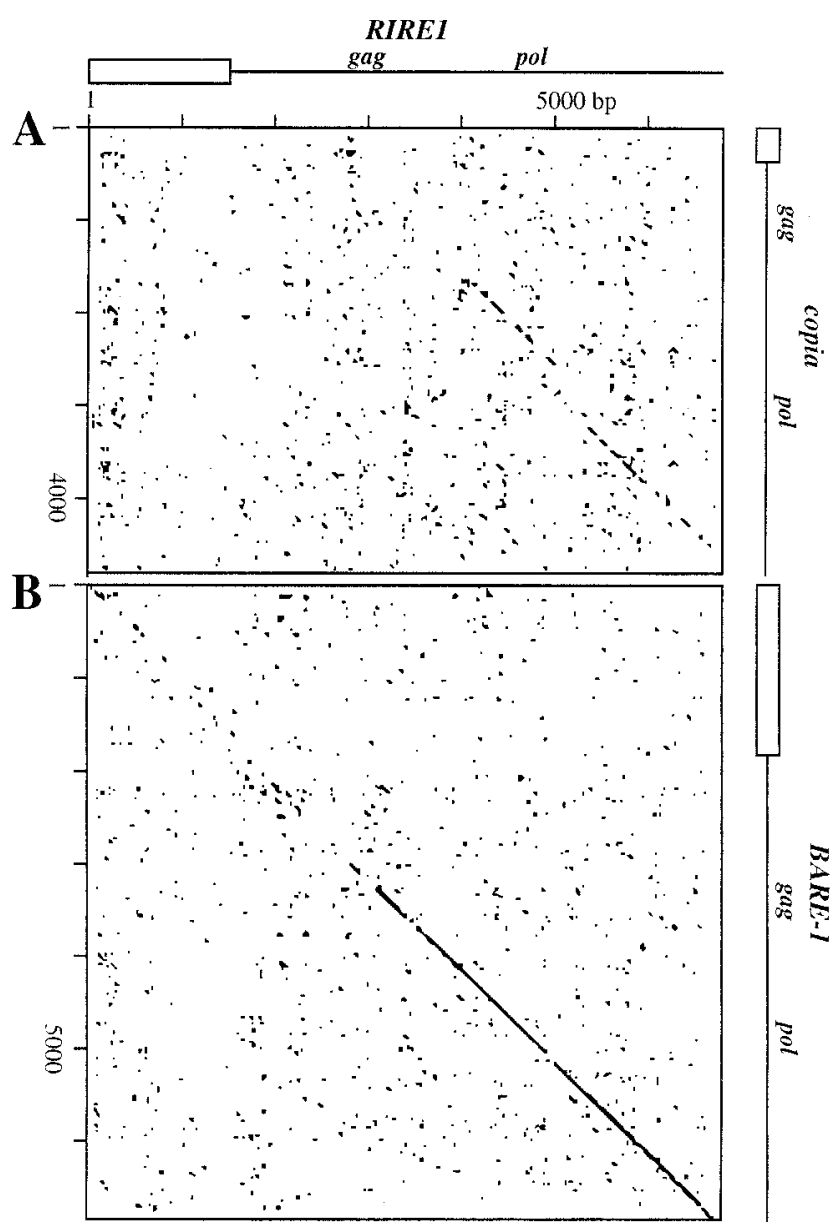

Fig. 5. Dot matrix between the nucleotide sequences of RIRE1 and copia $(\mathbf{A})$ or BARE-1 (B). Dots are placed at locations of the identical nucleotides when more than 25 nucleotides out of 50 are identical. Structures of retrotransposons with LTR at the 5' end and the internal region are shown at top and on the right side. with a zinc-finger motif, which is supposed to be involved in DNA binding (Johnson et al., 1986). The coding region corresponding to int existed in RIRE1 at positions 41235022 (Fig. 4) and contained this zinc-finger motif (Fig. 6). Besides the zinc-finger motif, another motif D, D (35) E involving in processes of integration is conserved in integrases encoded by retroelements (Khan et al., 1991; Kulkosky et al., 1992; Doak et al., 1994). This motif was also present in int in RIRE 1 (Fig. 6). The $r t$ gene encodes a reverse transcriptase which is generally conserved well in many retroelements. Site-specific mutagenesis in the amino acid sequence Y-X-D-D in the conserved region has decreased the reverse transcriptase activity severely, indicating that the sequence is one of the active sites (Lader et al., 1987). In RIRE1, the coding region corresponding to $r t$ existed at positions 5023-6288 (Fig. 4), and contained this sequence (Fig. 6). The $r h$ gene encodes RNase $\mathrm{H}$ which contains the $\mathrm{D}$ (34) E (19) D (51) D motif which is involved in metalbinding (Davies et al., 1991). The coding region corresponding to this gene in RIRE1 existed at positions 62896777 (Fig. 4), and contained this motif (Fig. 6).

The structure of LTR. As described above, we determined the sequence of LTR of RIRE 1 to be $1523 \mathrm{bp}$ (Figs. 2 and 4). Retrotransposons and retroviruses contain two LTR sequences almost identical each other at their ends. The LTR sequence determined in this study is the consensus sequence of those derived from two LTRs which are supposed to be present in RIRE1 as shown in Figure 4.

The size of LTR in RIRE1 was similar to those in two plant retrotransposons of copia type, BARE-1 in barley (Manninen and Schulman, 1993) and Wis-2-1A in wheat (Moore et al., 1991; Lucas et al., 1992; Murphy et al., 1992), which carry long LTR of 1829 and $1755 \mathrm{bp}$, respectively. The LTR sequence in RIRE1, however, showed poor homology with those of BARE-1 and Wis-2-1A (less than 50\%), although the internal region showed good homology with those in the retrotransposons (see Fig. 5B for the homologous relationship between RIRE1 and BARE-1). Mouse mammary tumor virus (MMTV) carries a long LTR, 1330
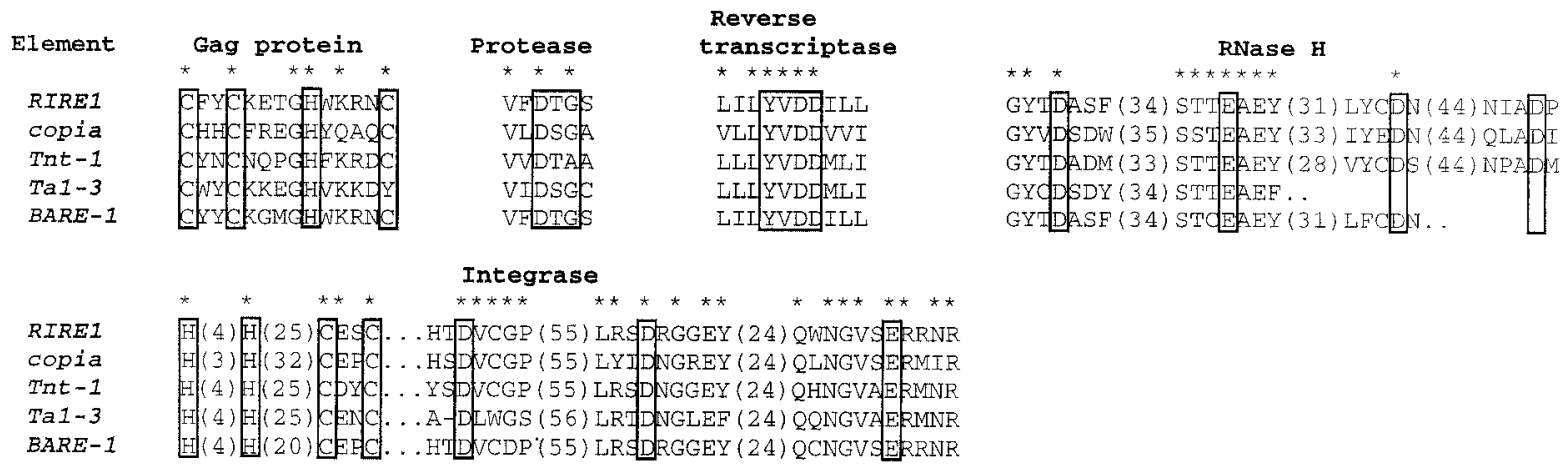

Fig. 6. Amino acid sequences containing motifs or particular sequences conserved in the proteins encoded by retrotransposons. Amino acids boxed indicate those important in motifs or sequences. Asterisks indicate amino acids conserved in more than 4 retrotransposons. 
bp in length, but this LTR did not show any homology with LTR of RIRE1.

LTR of retrotransposons are divided into three functional regions, U3, R, and U5 (Temin, 1981, Birnstiel et al., 1985; Varmus, 1983): The U3 region contains a promoter for RNA polymerase II, and the $\mathrm{R}$ region is defined to be the region from a cap site to a polyadenylation site; the U5 region is defined to be the region downstream of the polyadenylation site. It has been pointed out that variations in size occur in the U3 region in retroviruses (Temin, 1981). LTR of RIRE 1 contained sequence CCACT (see Fig. 4) at positions 1053-1057, which is a promoter sequence in Rous sarcoma virus (RSV) (Swanstrom et al., 1981). Another promoter sequence, TATAA, was present in the region at positions 1079-1083, 19 bp downstream of the CCACT sequence in RIRE1 (Fig. 4). The termination signal, TTGT, for retroviral RNA synthesis (Temin 1981) existed at positions 1334-1487 in the LTR sequence of RIRE1 (see Fig. 4).

Insertion of one RIRE1 member into another. As described earlier, PCR using a pair of primers, which hybridize to the end regions of LTR and prime DNA synthesis towards outside of the sequence, produced the fragments containing a portion of RIRE 1 due to the presence of many RIRE1 members with an insertion of another RIRE1 member (see Fig. 1). We investigated the insertion sites of RIRE1 further by PCR using several pairs of primers, which hybridize to either end region of LTR and with a region inside of LTR (Fig. 7). Electrophoresis of the PCR products in a polyacrylamide gel and sequencing of some of them could locate many insertion sites in LTR of RIRE1 (Fig. 7). We could also locate the insertion sites of RIRE 1 in the internal region of RIRE1 by analyzing the PCR products using primers which hybridize to an end region of LTR and to a region in the internal region (data not shown). The insertion sites determined do not seem to be clustered in a particular site or region (see Fig. 7).

\section{DISCUSSION}

In this paper, we have determined the entire nucleotide sequence of RIRE1, and shown that RIRE1 is a typical copia-like retrotransposon. RIRE1 becomes the first retrotransposon of rice whose entire sequence has been determined. A clone (pRN134; Fig. 2) screened from a genomic DNA library contained solo LTR which was flanked by direct repeats of a 5-bp sequence. It is known that retroposition of retrotransposons and retroviruses generates a duplication of a target site sequence of several bp in length (Bingham and Zachar, 1989). Since solo LTR is supposed to be produced by recombination between two LTR sequences present at the ends of retrotransposons (Roeder and Fink, 1980), the result above indicates that RIRE1 generates a duplication of a 5-bp sequence at the target upon retroposition.

We have described here that RIRE1 has a long LTR se-

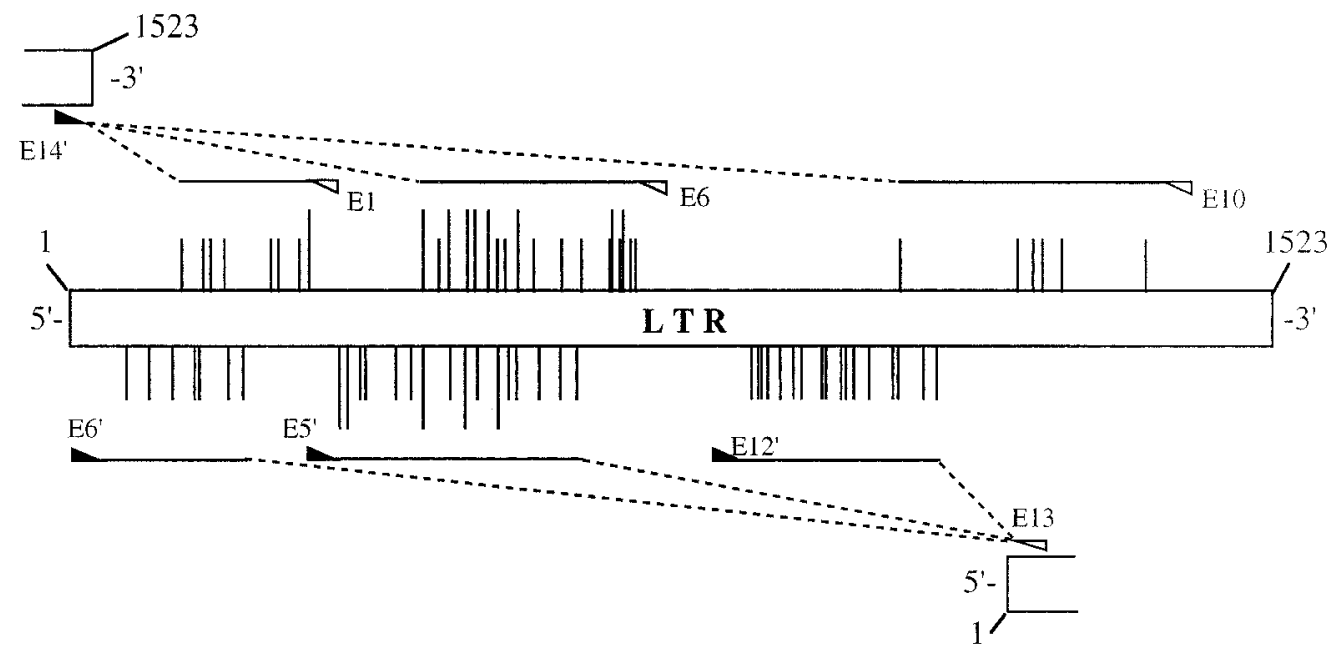

Fig. 7. Insertion sites of RIRE1 in the LTR sequence of RIRE1. Short vertical bars indicate insertion sites mapped by lengths of the PCR-amplified fragments which were determined by gel electrophoresis. Long vertical bars indicate insertion sites mapped by cloning and sequencing of the PCR-amplified fragments. The PCR fragments were amplified using primer E14', which hybridizes to the 3'-end region of LTR and primes DNA synthesis towards the outside of LTR, and the other primer, such as E1, E6 or E10 (open arrowheads), which hybridizes to the sequence inside of LTR and primes DNA synthesis from right to left (see Table 1). These fragments were used to map the insertion sites shown above the structure of LTR. The PCR fragments were also amplified using primer E13, which hybridizes to the 5' end region of LTR and primes DNA synthesis towards outside of LTR, and the other primer, such as E5' E6' or E12' (solid arrowheads), which hybridizes to the inside sequence of LTR and primes DNA synthesis from left to right (see Table 1). These fragments were used to map the insertion sites shown below the structure of LTR. Horizontal lines with an arrowhead indicate the largest PCR fragments analyzed. 
quence like BARE-1 in barley and Wis-2-1A in wheat. LTR of RIRE1 shows poor homology with those of BARE-1 and Wis-2-1A, although those in the latter retrotransposons are highly homologous each other (Manninen and Schulman, 1993). In the LTR sequence of Wis-2-1A, there exist many direct and inverted repeats of short DNA sequences. It is supposed that genetic alteration caused by an imprecise excision of a transposon happened to be inserted into LTR of Wis-2-1A or slippage occurring during reverse transcription have elongated the LTR sequence (Lucas et al., 1992). RIRE1 also contained many direct and inverted repeats like Wis-2-1A, suggesting that the same kinds of events described above might have occurred in an ancestor retrotransposon of RIRE1 to lengthen its LTR.

Retroposition of retrotransposon Tnt 1 in tobacco is activated by treatment with cell wall hydrolases used to release protoplasts (Pouteau et al., 1991). Retroposition of retrotransposon Tos 17 in rice is activated during culturing and regeneration of rice tissues (Hirochika et al., 1996). To see whether retroposition of RIRE1 is activated during culturing and regeneration of tissues of $O$. sativa like Tos 17 , we carried out Southern hybridization analysis of total DNA prepared from the regenerated rice plants from protoplasts, and observed no extra bands attributed to retroposition of RIRE1 (H. Hirochika, H. Ohtsubo and E. Ohtsubo, unpublished results). From this experiment, however, we cannot determine whether RIRE1 is activated or not, since the $O$. sativa genome contains about 180 copies of RIRE 1 (Nakajima et al., 1996), and the new bands, even if appeared, might be overlapped with the bands of the fragments derived from each copy of RIRE1 preexisted in the rice genome.

The LTR sequences of RIRE 1 are present in O. australiensis in about 7500 copies per haploid genome (Nakajima et al., 1996). In this paper, we have shown that there existed many RIRE1 members with an insertion of another RIRE1 member. This indicates that these RIRE1 members have received another RIRE1 member to make an extraordinary number of copies of it in the $O$. australiensis genome without giving a deleterious effect on the growth of rice cells. Many retrotransposons isolated in eukaryotes are inserted at a specific region, such as the $5^{\prime}$ regions of genes (for a review, see Sandmeyer et al., 1990). We do not know at present whether RIRE1 is also inserted in the 5 ' region of genes or not. We know, however, that RIRE1 is not inserted into any particular sites in the LTR sequence of itself. We know also that RIRE 1 resides throughout the chromosomes in O. australiensis as revealed from a study of fluorescence in situ hybridization (FISH) using LTR of RIRE1 as a probe (Ohmido, Fukui, E. Ohtsubo and H. Ohtsubo, unpublished results).

The results of flow cytometry indicate that the size of the haploid genome of $O$. sativa is $434 \mathrm{Mb}$, while the size of O. australiensis is $960 \mathrm{Mb}$, about 2 times larger than that of $O$. sativa (Martinez et al., 1994). The number of copies of LTR of RIRE1 in the haploid genome of O. sativa is about 180 , while it is 7500 in O. australiensis (Nakajima et al., 1996). This indicates that RIRE1 caused the increase of size of $11 \mathrm{Mb}$ (if all the LTRs are present as solo LTR) or 31 $\mathrm{Mb}$ (if all the LTRs are portions of RIRE1 with the internal region) in O. australiensis. It is, therefore, considered that the increase of RIRE 1 copies has contributed in part to the increase of size of the genome of $O$. australiensis.

We thank Y. Sano (National Institute of Genetics) for providing us seeds of the rice strain used in this study. We also thank our colleague Y. Sekine for valuable discussion. This work was supported by a Grant-in Aid for Scientific Research from Ministry of Education, Science, Sports, and Culture of Japan, and by a grant Pioneering Project in Biotechnology from the Ministry of Agriculture, Forestry, and Fishery of Japan.

\section{REFERENCES}

Berg, J. M. (1986) Potential metal-binding domains in nucleic acid binding proteins. Science 232, 485-487.

Bingham, P. M. and Zachar, Z. (1989) Retrotransposons and the FB transposon from Drosophila melanogaster: In: Mobile DNA (eds.: D. E. Berg and M. M. Howe), pp. 485-502. American Society for Microbiology, Washington, DC.

Birnstiel, M. L., Busslinger, M., and Strub, M. (1985) Transcription termination and 3 ' processing: The end is in site! Cell 41, 349-359.

Davies, J. F., Hostomska, Z., Hostomsky, Z., Jordan, S. R., and Matthews, D. A. (1991) Crystal structure of the ribonuclease H domain of HIV-1 reverse transcriptase. Science 252, 8895.

Doak, T. G., Doerder, F. P., Jahn, C. L., and Herrick, G. (1994) A proposed superfamily of transposase genes: Transposon-like elements in ciliated protozoa and a common "D35E" motif. Proc. Natl. Acad. Sci. USA 91, 942-946.

Flavell, A. J., Dunbar, E., Anderson, R., Pearce, S. R., Hartley, R., and Kumar, A. (1992) Ty1-copia group retrotransposons are ubiquitous and heterogeneous in higher plants. Nucleic Acids Res. 20, 3639-3644.

Grandbastien, M.-A., Spielmann, A., and Caboche, M. (1989) Tnt1, a mobile retroviral-like transposable element of tobacco isolated by plant cell genetics. Nature 337, 376-380.

Hirochika, H., Fukuchi, A., and Kikuchi, F. (1992) Retrotransposon families in rice. Mol. Gen. Genet. 233, 209-216.

Hirochika, H. and Hirochika, R. (1993) Ty1-copia group retrotransposons as ubiquitous components of plant genomes. Jpn. J. Genet. 68, 35-46.

Hirochika, H., Sugimoto, K., Otuki, Y., Tsugawa, H., and Kanda, M. (1996) Retrotransposons of rice involved in mutations induced by tissue culture. Proc. Natl. Acad. Sci. USA 93, 77837788 .

Johnson, M. S., McClure, M. A., Feng, D.-F., Gray, J., and Doolittle, R. F. (1986) Computer analysis of retroviral pol genes: Assignment of enzymatic functions to specific sequences and homologies with nonviral enzymes. Proc. Natl. Acad. Sci. USA 83, 7648-7652.

Khan, E., Mack, J. P., Katz, R. A., Kulkosky, J., and Skalka, A. M. (1991) Retroviral integrase domains: DNA binding and the recognition of LTR sequence. Nucleic Acids Res. 19, 851-860.

Kulkosky, J., Jones, K. S., Katz, R. A., Mack, J. P. G., and Skalka, A. M. (1992) Residues critical for retroviral integrative recombination in a region that is highly conserved among retroviral/ 
retrotransposon integrases and bacterial insertion sequence transposases. Mol. Cell. Biol. 12, 2331-2338.

Lader, B. A., Purifoy, D. J. M., Powell, K. L., and Darby, G. (1987) Site-specific mutagenesis of AIDS virus reverse transcriptase. Nature 327, 716-717.

Lapatto, R., Blundell, T., Hemmings, A., Overington, J., Wilderspin, A., Wood, S., Merson, J. R., Whittle, P. J., Danley, D. E., Geoghegan, K. F., Hawrylik, S. J., Lee, S. E., Scheld, K. G., and Hobart, P. M. (1989) X-ray analysis of HIV-1 proteinase at 2.7 $\AA$ resolution confirms structural homology among retroviral enzymes. Nature 342, 299-302.

Lee, D., Ellis, T. H. N., Turner, L., Hellens, R. P., and Cleary, W. G. (1990) A copia-like element in Pisum demonstrates the uses of dispersed repeated sequences in genetic analysis. Plant Mol. Biol. 15, 707-722.

Lucas, H., Moore, G., Murphy, G., and Flavell, R. B. (1992) Inverted repeats in the long-terminal repeats of the wheat retrotransposon Wis-2-1A-A. Mol. Biol. Evol. 9, 716-728.

Manninen, I. and Schulman, A. H. (1993) BARE-1, a copia-like retroelement in barley (Hordeum vulgare L.). Plant Mol. Biol. 22, 829-846.

Martinez, C. P., Arumuganathan, K., Kikuchi, H., and Earle, E. D. (1994) Nuclear DNA content of ten rice species as determined by flow cytometry. Jpn. J. Genet. 69, 513-523.

Messing, J. (1983) New M13 vectors in cloning. Methods Enzymol. 101, 20-78

Moore, G., Lucas, H., Batty, N., and Flavell, R. B. (1991) A family of retrotransposon and associated genomic variation in wheat. Genomics 10, 461-468.

Murphy, G. J. P., Lucas, H., Moore, G., and Flavell, R. B. (1992) Sequence analysis of WIS-2-1A, a retrotransposon-like element from wheat. Plant Mol. Biol. 20, 991-995.

Nakajima, R., Noma, K., Ohtsubo, H., and Ohtsubo, E. (1996) Identification and characterization of two tandem repeat sequences (TrsB and TrsC) and a retrotransposon (RIRE1) as genomegeneral sequences in rice. Genes Genet. Syst. 71, 373-382.

Ohtsubo, H., Umeda, M., and Ohtsubo, E. (1991) Organization of DNA sequences highly repeated in tandem in rice genomes. Jpn. J. Genet. 66, 241-254.

Pearl, L. H. and Taylor, W. R. (1987) A structural model for the retroviral proteases. Nature 329, 351-354.

Pouteau, S., Huttner, E., Grandbastien, M. A., and Caboche, M. (1991) Specific expression of the tobacco Tnt1 retrotransposon in protoplasts. EMBO J. 10, 1911-1918.

Prats, A. C., Sarih, L., Gabus, C., Litvak, S., Keith, G., and Darlix, J. L. (1988) Small finger protein of avian and murine retroviruses has nucleic acid annealing activity and positions the replication primer tRNA onto genomic RNA. EMBO J. 7, $1777-1783$

Roeder, G. S. and Fink, G. R. (1980) DNA rearrangements associated with a transposable element in yeast. Cell 21, 239-249.

Sambrook, J., Fritsch, E. F. and Maniatis, T. (1989) Molecular Cloning: A Laboratory Manual, 2nd ed. Cold Spring Harbor Laboratory, Cold Spring Harbor, NY.

Sandmeyer, S. B., Hansen, L. J., and Chalker, D. L. (1990) Integration specificity of retrotransposon and retroviruses. Annu. Rev. Genet. 24, 491-518.

Sanger, F., Nicklen, S., and Coulson, A. R. (1977) DNA sequencing with chain-terminating inhibitors. Proc. Natl. Acad. Sci. USA 74, 5463-5467.

Skalka, A. M. (1989) Retroviral proteases: First Glimpses at the anatomy of a processing machine. Cell 56, 911-913.

Smyth, D. R., Kalitsis, P., Joseph, J. L., and Sentry, J. W. (1989) Plant retrotransposon from Lilium henryi is related to Ty3 of yeast and the gypsy group of Drosophila. Proc. Natl. Acad. Sci. USA 86, 5015-5019.

Swanstrom, R., Delorbe, W. J., Bishop, J. M., and Varmus, H. E. (1981) Nucleotide sequence of cloned unintegrated avian sarcoma virus DNA: Viral DNA contains direct and inverted repeats similar to those in transposable elements. Pro. Natl. Acad. Sci. USA 78, 124-128.

Temin, H. M. (1981) Structure, variation and synthesis of retrovirus long terminal repeat. Cell 27, 1-3.

Tenzen, T., Matsuda, Y., Ohtsubo, H., and Ohtsubo, E. (1994) Transposition of Tnr1 in rice genomes to 5'-PuTAPy-3', duplicating the TA sequence. Mol. Gen. Genet. 245, 441-448.

Varmus, H. E. (1983) Retroviruses. In: Mobile Genetic Elements (ed.: I. A. Shapiro), pp. 411-503. Academic Press, New York.

Varmus, H. and Brown, P. (1989) Retroviruses. In: Mobile DNA (ed.: D. E. Berg and M. M. Howe), pp. 53-108. American Society for Microbiology, Washington, DC.

Vieira, J. and Messing, J. (1987) Production of single stranded plasmid DNA. Methods Enzymol. 153, 3-11.

Voytas, D. F. and Ausubel, F. M. (1988) A copia-like transposable element family in Arabidopsis thaliana. Nature 336, 242244.

Voytas, D. F., Cummings, M. P., Konieczny, A., Ausubel, F. M., and Rodermel, S. R. (1992) Copia-like retrotransposons are ubiquitous among plants. Proc. Natl. Acad. Sci. USA 89, 71247128.

Zhao, X., Wu, T., Xie, Y., and Wu, R. (1989) Genome-specific repetitive sequences in the genus Oryza. Theor. Appl. Genet. 78, 201-209. 